\title{
RESOURCE ELEMENT-LEVEL DIMENSIONING OF LONG TERM EVOLUTION NETWORKS
}

\author{
Arshed Oudah \\ Faculty of Manufacturing Engineering \\ Universiti Malaysia Pahang \\ 26600, Pekan, Pahang \\ Corresponding author: arshed.ump@gmail.com ${ }^{I}$
}

\begin{abstract}
Long Term Evolution (LTE) of Universal Mobile Telecommunications System (UMTS) is the latest cellular technology coined by the third Generation Partnership Project (3GPP) and is currently being deployed worldwide. a crucial step towards the successful deployment scenarios of LTE networks is through the use of accurate budgetary and coverage dimensioning formulas of LTE's physical air-interface channels and signals to guarantee favourable cell footprint and other capacity targets. In this paper, the total physical downlink resources along with their associated powers are derived and computed. Their corresponding overheads are also presented. The provided formulas serve in various network dimensioning campaigns of the LTE systems.
\end{abstract}

Keywords: Long term evolution, dimensioning, link budget, resource element, control channels, overhead, cell physical resources

\section{INTRODUCTION}

After being ratified by the International Telecommunication Union (ITU) as the fourth generation $(4 \mathrm{G})$ technology, The $3^{\text {rd }}$ Generation Partnership Project (3GPP) long term evolution of Universal Mobile Telecommunications System (UMTS) ${ }^{1}$ (Kattan, 2009) has broken all records as the fastest cellular technology being embraced and deployed worldwide (The Global mobile Suppliers Association (GSA), 2011a, 2011b, 2011c, 2011d).

LTE is also referred to as Evolved UMTS Terrestrial Radio Access Network (E-UTRAN) 
Based on the specifications (3GPP TS 25.942, 2008; 3GPP TS 36.211, 2007; 3GPP TS 36.300, 2006; 3GPP TS 36.410, 2010), LTE air-interface gives rise to large transmission bandwidths of $1.4 \mathrm{MHz}$ up to $20 \mathrm{MHz}$ in which several physical common ${ }^{2}$ control channels, synchronization and pilot signals are transmitted and received along with user data. Similar to UMTS (3GPP TS $25.211,2002$; 3 GPP TS 25.221, 2002), these channels and signals profoundly decide cell coverage and capacity targets. Nevertheless, owing to LTE-toUMTS bandwidth and data rate ratios, their overhead and power requirements are quite different from those of UMTS, i.e. they have larger powers, and therefore precise dimensioning of the channels and signals becomes extremely imperative and decisive for efficient intake of cell power and physical resources as well as controlled coverage and capacity targets.

Notably, the physical layer resources and its corresponding overhead analysis are poorly covered in the standard (3GPP TS 36.211, 2007). Nonetheless, dimensioning of downlink physical channels and signals are neither sufficiently nor efficiently covered.

The objective of this manuscript is to address these limitations in the standard in the related literature by proposing accurate formulas for the calculations of downlink channels and signal resource elements numbers of an LTE evolved Node-B (eNodeB), their associated overhead and their corresponding power intakes before and after reception. Furthermore, to accurately account for the various clutter types and variable cell range, an improved propagation model is proposed for the modelling of physical channels received powers. The finding of this work can thus be efficiently used to bolster up cell coverage and capacity campaigns for a wide range of transmission possibilities.

\section{RELATED WORK}

This section aims at leveraging reader's insights on latest research activities, and specifically, the ways they are employed to characterize and model physical cell resources in OFDMA-centric systems particularly in LTE networks.

At the heart of any eNodeB, the physical layer and air-interface schemes and their corresponding control channels and signals (RS, $\mathrm{PBCH}, \mathrm{SS}, \mathrm{PDCCH}$, and $\mathrm{PDSCH}$ ) are the main drivers of the whole transmission process and thus, are the controllers of coverage, capacity and actual cell throughput in LTE (3GPP TS 36.211, 2007). They are therefore, the key elements in many decisive LTE dimensioning processes.

2 Unlike legacy systems, LTE is a packet-switched standard; therefore only common-type channels are supported. Dedicated channels are no more required. 
For these reasons and many others, there has been a wave of research activities dedicated to the design and characterization of LTE's PHY layer channels and signals.

Unfortunately, the $3 \mathrm{GPP}$ does not provide a complete modelling procedures of the LTE's frame structure and its built-in control channels (3GPP, 2007; 3GPP, 2008). A natural extension of this shortage is a wide spectrum of inputs from the literature filling in the gaps left by the standard.

Accordingly, the authors, Ball, Hindelang and Kambourov (2008) provided brilliant design and performance comparisons between LTE and its rival WiMAX. After exposing a complete picture of the PHY layer channels and signals of both technologies, they illustrated that the overall radio performance of both systems is similar, with LTE slightly outperforming WiMAX. The authors albeit their in-depth treatment, provided no models to account for the channels resource utilization or inputs pertaining to channel overhead except for Reference signal (RS) and Physical Download Control Channel (PDCCH). In the same context, the work by Cui (2009) validated the results in the standard and went beyond the theory of LTE by concluding that the new protocol's practical DL/UL data rates are only (257/41) Mbps, as opposed to $(300 / 71)$ Mbps in theory, provided that of $20 \mathrm{mhz}$ of bandwidth with 4x4 mimo antenna configurations are used. This paper does not include resource elements calculations, and the simulated system was limited to FDDmode only.

In contrast to Cui (2009), the investigation by Rezaei, Hempel and Sharif (2011) revealed that LTE's (Rel.8.0) DL-to-UL speed is (300-to- 75) Mbps. The results were collected by the modelling and simulation of LTE-PHY layer system operating in both FDD and TDD modes. The study, however, is shortened by the lack of control of channels overhead.

Similarly, the findings of Basit (2009), Ratasuk, Ghosh, Love, Nory and Classon (2008), Rezaei (2010), Sanchez, Morales-Jimenez, Gomez and Enbrambasaguas (2007) are of similar nature. They provided some insights into some theoretical performance metrics of LTE. Nevertheless, apart from the impractical assumptions they relied on in their studies, the provision of no analytical models of calculating resource elements and overheads were the common features noted in these studies.

\section{RESEARCH METHODOLOGY}

Figure 1 depicts A Long Term Evolution (LTE) signal flow diagram in which the physical download resources along with their associated phases 
of transmission and reception are proposed. Interestingly, the methodology employed can BE basically broken down into the following:

- $\quad$ The transmission power of LTE frame resource element is calculated first. This power is determined in line with the transmission power of LTE base station antenna port;

- $\quad$ Matlab ${ }^{\circledR}$ programming language is used as the simulation tool, where the parameters in Table 1 along with formulas in section 4, 5 and 6 are used together to determine the LTE downlink signals and channel powers.

Interestingly, three mediums have been defined in Figure 1: transmitter, transmission medium and receiver. The diagram is further subdivided using points A-to-S, which include various factors affecting the transmission and reception of the LTE downlink frame (together with its signals and channels).

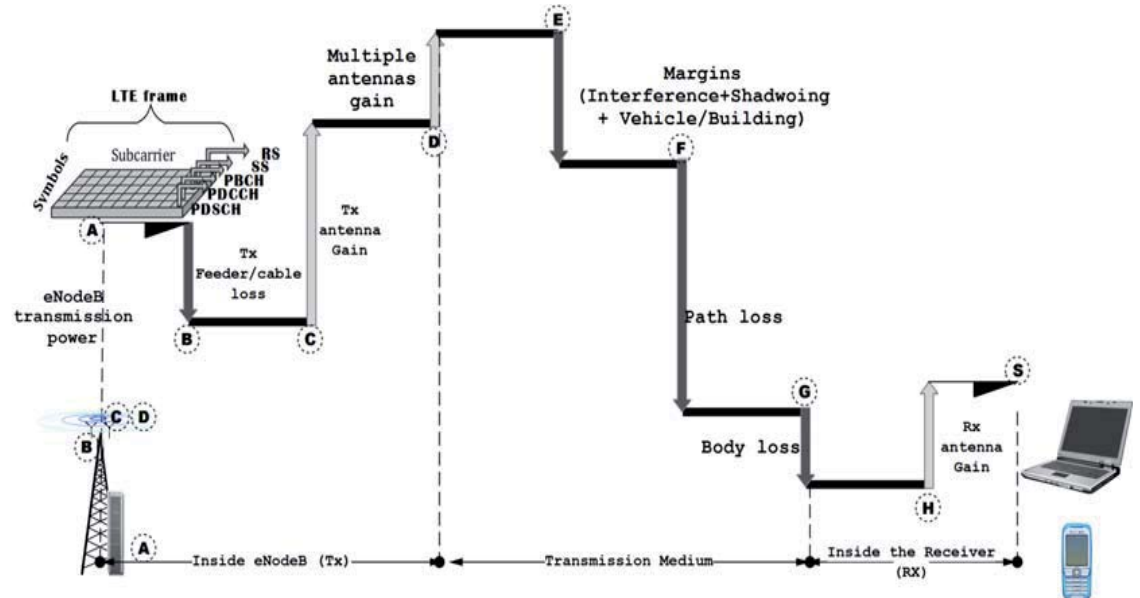

Figure 1. Calculation-diagram for downlink signals and channels powers path between LTE eNodeB and receiver.

In this regard, several Matlab ${ }^{\circledR}$ simulation runs are performed to evaluate the performance of an LTE eNodeB. Effects of the common channels and signals overhead on traffic channels and the use of multiple transmission antenna ports at the base station for various channel bandwidths are investigated together. 


\section{Table1}

\section{Simulation Parametes}

\begin{tabular}{lclc}
\hline \multicolumn{1}{c}{ Parameter } & Value & \multicolumn{1}{c}{ Parameter } & Value \\
\hline eNodeB power $(\mathrm{dBm})$ & 46 & Interference margin $(\mathrm{dB})$ & 3 \\
No. of Tx antennas & $1,2 \& 4$ & Feeder and jumper losses $(\mathrm{dB})$ & 0.4 \\
Tx antenna gain $(\mathrm{dBi})$ & 18 & Tx antenna height $(\mathrm{m})$ & 30 \\
Shadowing margin $(\mathrm{dB})$ & 6 & Rx antenna height $(\mathrm{m})$ & 1.5 \\
Feeder + Jumper loss $(\mathrm{dB})$ & 0.2 & Operating frequency $(\mathrm{MHz})$ & 1800 \\
Body loss $(\mathrm{dB})$ & 0 & Transmitter bandwidth $(\mathrm{MHz})$ & 1.4 \\
Penetration loss $(\mathrm{dB})$ & 18 & Tx to Rx distance $(\mathrm{m})$ & 800 \\
Control region $(\mathrm{sym})$ & 2 & & \\
\hline
\end{tabular}

\section{RESOURCE ELEMENT TRANSMISSION POWER}

An LTE eNodeB transmits power that is shared equally by all antenna transmission ports. the Antenna port power is then used to feed the transmission of a frame by sharing its power with all physical downlink resource elements. Analytically, the following holds true for any number of antenna ports supported by an eNodeB:

$$
P_{a n t}=\frac{P_{e N o d e B}}{N_{a n t}}
$$

Where $P_{\text {ant }}$ is the antenna port maximum transmit power in Watt, $P_{\text {eNodeB }}$ is the maximum transmit power of an eNodeB in Watt and $N_{a n t}$ is the number of antenna ports supported by the same cell. In LTE release 8.0, which is currently being deployed worldwide, $P_{a n t}$ is equally divided over frame resource elements:

$$
P_{r e}=\frac{P_{a n t}}{N_{r e}^{\text {frame }}}
$$

Where $P_{r e}$ is the power of a single resource element, $P_{a n t}$ is found in Eq.(1), and is the total number of RE(s) in a frame and $N_{r e}^{\text {frame }}$ is expressed as follows:

$$
N_{r e}^{\text {frame }}=12 \times 20 \times N_{\text {Sym }}^{\text {slot }} \times N_{R B}
$$

Where 12 is the number of subcarriers in one timeslot, 20 is the number of timeslots per one frame, $N_{S y m}^{\text {slot }}$ is the number of OFDM symbols in one timeslot, which is 7 or 6 for normal or extended cyclic prefix, respectively, $N_{R B}$ is 
the number of resource blocks in one frame, which ranges from 6 to 10 for $1.4 \mathrm{MHz}$ to $20 \mathrm{MHz}$, respectively. Figure 2 depicts a $1.4 \mathrm{MHz}$ LTE downlink frame configuration along with its associated physical channels and signals.

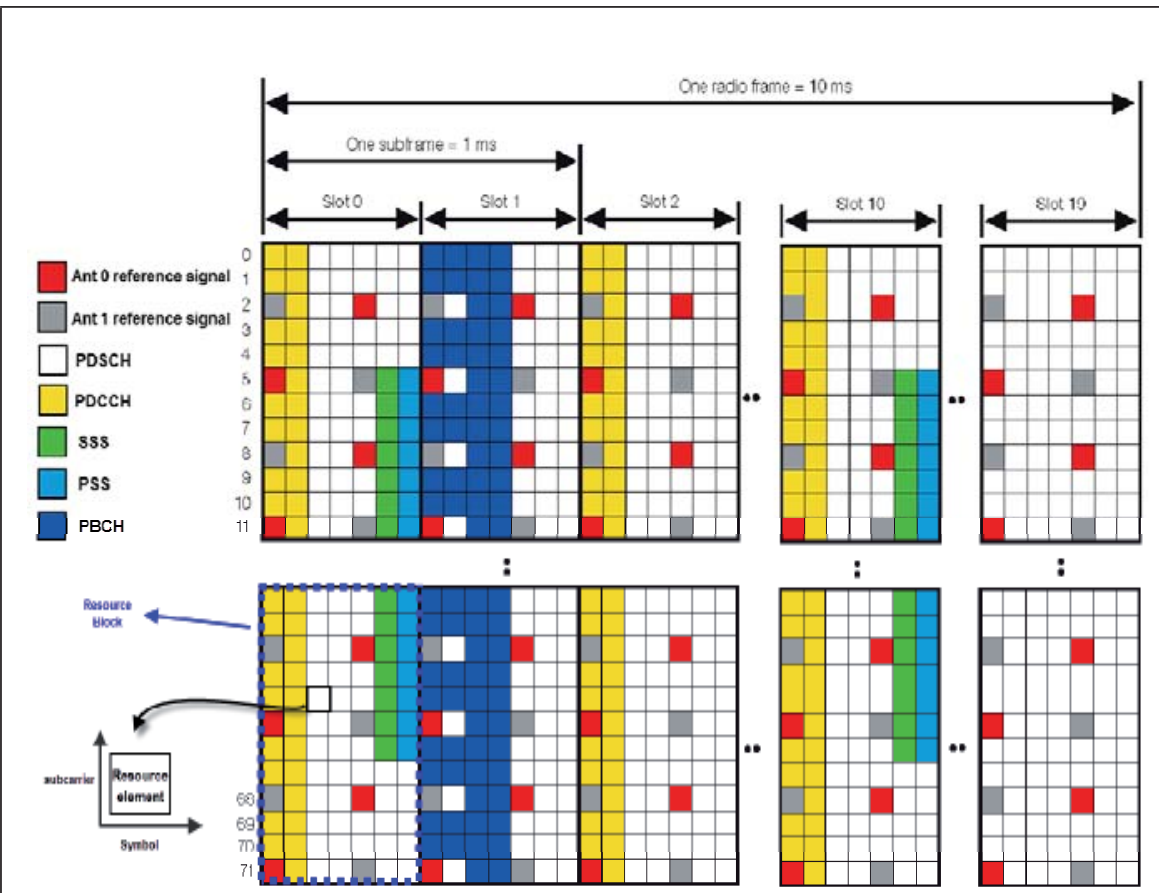

Figure 2. LTE frame structure of $1.4 \mathrm{MHz}$ bandwidth and two antenna ports.

\section{LTE PHYSICAL DOWNLINK CHANNELS AND SIGNALS POWERS}

This section embarks on the formulation of physical downlink channels and signals powers, as illustrated in Figure 1. The objective here is to derive power formulas that are applicable to any Frequency Division Multiplexing (FDD) type of frame in any LTE system and for a wide range of system-specific parameters. interestingly, the same formulas can be used for the time division multiplexing (TDD) type of frame; however, some additional frame-type constraints found in 3gpp ts 36.211 (2007) have to be respected. To end of this work, Figure 2 is employed in which two major phases, namely, points a and $\mathrm{s}$ are used for demonstration purposes. These phases, i.e. a and s, correspond to transmission and reception respectively. 


\section{Power at point $A$}

Point $\mathrm{A}$ is the point where the resource elements are assigned to their perspective physical channels and signals, i.e. They are powered up. However, at this point no signal attenuation is undergone as shown in Figure 2.

Power models of physical channels and signals at point A are as follows:

a) Downlink Reference Signals

At point A in Figure 2, reference signal (RS) power $P_{R S}^{T x}$ is the power of RS prior to any loss. This power (in Watt) can be expressed as follows:

$$
P_{R S}^{T x}=P_{r e} \times\left(N_{r e-u s e d}^{R S}+N_{r e-s i l e n t}^{R S}\right)
$$

Where $P_{r e}$ is the resource element power (in Watt) as found in Eq.(2), $N_{r e-u s e d}^{R S}$ and $N_{r e-s i l e n t}^{R S}$ is the number of reference signals used and silent resource elements, respectively in one frame and are found as follows:

In one timeslot, there are 4 used reference signals resource elements for 1 and 2 transmit antenna ports respectively, and $6 \mathrm{RE}(\mathrm{s})$ for the case of 4 ports. However, unlike used resource elements, there are 0,4 or 8 silent ones for 1 , 2 or 4 antenna ports respectively. For per frame, this gives:

$$
\begin{aligned}
& N_{\text {re-used }}^{R S}=20 \times 4 \times N_{R B} \text { For } 1 \text { or } 2 \text { transmit antenna ports } \\
& \text { Or } \quad N_{\text {re-used }}^{R S}=20 \times 6 \times N_{R B} \quad \text { For } 4 \text { transmit antenna ports }
\end{aligned}
$$

Where $(20 \times 4)$ is the number of RS used resource elements per one resource block and $N_{R B}$ is the number of resource blocks per a frame.

Similarly, number of silent resource elements can be found as below:

$$
\begin{array}{cc}
N_{r e-s i l e n t}^{R S}=0 & \text { For 1 antenna port } \\
N_{r e-\text { silent }}^{R S}=20 \times 4 \times N_{R B} & \text { For 2 antenna port } \\
\text { Or } \quad N_{r e-\text { silent }}^{R S}=20 \times 8 \times N_{R B} & \text { For 4 antenna port }
\end{array}
$$




\section{b) Downlink Synchronization Signals}

The mapping of the Primary Synchronization Signals (PSS) and Secondary Synchronization Signals (SSS) resource elements is bandwidth-independent as they are only transmitted through the middle six resource blocks. Accordingly, the number of resource elements per each synchronization signal is 144, which gives a total of 288 for both synch signals. The power (in linear units) of synchronization signals (SS) is thus:

$$
P_{s s}^{T x}=P_{r e} \times 288
$$

Where $P_{r e}$ is the power (W) of single resource element, as found in Eq.(2).

c) Physical Broadcast Channel

The Physical Broadcast Channel (PBCH) takes up 4 OFDM symbols in the second timeslot of the first downlink subframe, i.e. subframe \#0 in Figure 1. Additionally, $\mathrm{PBCH}$ resource elements overlap with those of reference signals and therefore have to be deducted as below:

$$
N_{r e}^{P B C H}=288-Y
$$

Where 288 is the number of $\mathrm{PBCH}$ resource elements in a frame and $Y$ denotes the number of RS overlapped resource elements, that is 12,24 or 48 for antenna ports number of 1,2 or 4 respectively. Accordingly, SS power $P_{s s}^{T x} \mathrm{i}$ is defined (in W) as:

$$
P_{s s}^{T x}=P_{r e} \times(288-Y)
$$

\section{d) Physical Downlink Control Channel}

The Physical Downlink Control Channel (PDCCH), Physical Hybrid ARQ Indicator Channel (PHICH) and Physical Control Format Indicator Channel $(\mathrm{PCFICH})$ together set up the control region in a frame and are mapped into one, two or three symbols of the first timeslot of any subframe. Like some other physical signals and channels, the control channels resource elements overlap with reference signals resource elements and thus have to be subtracted. Having known that, the following expression defines the number of resource elements used by the control region:

$$
N_{r e}^{\text {control }}=(12 \cdot s y m-K) \cdot 10 \cdot N_{R B}
$$


Where $N_{r e}^{\text {control }}$ is the number of resource elements taken up by control region, 12 represents the 12 subcarriers of a resource block, sym is the number of symbols occupied by the control region in one timeslot, $K$ represents/indicates the number of RS resource elements that overlap in the control region. It is thus:

$K=2$ (when 1 antenna port is configured),

$K=4$ (when 2 antenna ports are configured),

$K=4$ (when 4 antenna ports are configured and $s y m=1)$,

$K=8$ (when 4 antenna ports are configured and $s y m=2$ or 3 ),

$N_{R B}$ is the number of resource blocks per a frame (6 to 100) for $1.4 \mathrm{MHZ}$ to 20 $\mathrm{MHz}$, respectively and 10 is the number of subframes in one frame. From Eq. (8), control region power at point $\mathrm{A}$ is written (in Watt) as:

$$
P_{\text {control }}^{T x}=P_{r e} \times N_{r e}^{\text {control }}
$$

e) Physical Downlink Shared Channel

Whatever resource elements left vacant after all the physical downlink channels and signals have allocated their physical resources are used for the transmission of Physical Downlink Shared Channel (PDSCH) ${ }^{3}$, as expressed below:

$$
N_{r e}^{P D S C H}=N_{r e}^{\text {frame }}-\left(N_{r e}^{R S}+244+N_{r e}^{P B C H}+N_{r e}^{\text {control }}\right)
$$

Where $N_{r e}^{P D S C H}$ is the number of PDSCH resource elements in one frame, $N_{r e}^{\text {frame }}$ as found in Eq.(3), $N_{r e}^{R S}$ is the total RS (used plus silent) physical resources in a frame as found in subsection 5.1(a), 244, $N_{r e}^{P B C H}$, and $N_{r e}^{\text {control }}$ are the number of physical resources of synchronization signals, $\mathrm{PBCH}$ and control region respectively, as defined by subsections 5.1-(b),(c) and (d) respectively. Therefore, the downlink shared channel power at point A is expressed as follows:

$$
P_{P D S C H}^{T x}=P_{r e} \times N_{r e}^{P D S C H}
$$

\section{Power at point $S$}

Basically, point $\mathrm{S}$ is where an LTE frame is successfully received. This is considered true after the frame (including its associated physical channels and signals) has experienced a series of gains and losses across the transmissionreception path, i.e. from point $\mathrm{A}$ to $\mathrm{S}$ in Figure 2. Major gains and losses are: 
- $\quad$ Loss of cables, jumpers, feeder and tower mounted amplifier (TMA) insertion in $\mathrm{dB}$.

- $\quad$ eNodeB antenna gain which is proportional to antenna type and frequency band used.

- $\quad$ Multiple-Input-Multiple-output (MIMO) gain is the power boost owing to MIMO employment at the eNodeB.

- Margins or buffers to account for inevitable phenomena; such as intercell interference that pushes up the noise floor at the eNodeB due to adjacent cells and lognormal shadowing or shadow fading.

Attenuation due to transmission medium, i.e. Pathloss, which is propagation model-dependent outcome.

- $\quad$ Penetration loss due to penetrating building, vehicle, etc. This type of loss is frequency and morphology-dependent variable.

Body loss.

- Receiver antenna gain.

Accordingly, various physical signals and channels strength levels (in $\mathrm{dBm}$ ) can be expressed in the following way:

$$
\begin{aligned}
& P_{R S}^{R x}=P_{R S}^{T x}+G_{a n t}^{T x}+G_{M I M O}^{T x}-P L-M-L_{\text {building }}-L_{\text {Body }}+G_{\text {ant }}^{R x} \\
& P_{S S}^{R x}=P_{S S}^{T x}+G_{\text {ant }}^{T x}+G_{M I M O}^{T x}-P L-M-L_{\text {building }}-L_{\text {Body }}+G_{\text {ant }}^{R x} \\
& P_{P B C H}^{R x}=P_{P B C H}^{T x}+G_{\text {ant }}^{T x}+G_{M I M O}^{T x}-P L-M-L_{\text {building }}-L_{B o d y}+G_{\text {ant }}^{R x} \\
& P_{\text {control }}^{R x}=P_{\text {control }}^{T x}+G_{\text {ant }}^{T x}+G_{M I M O}^{T x}-P L-M-L_{\text {building }}-L_{\text {Body }}+G_{\text {ant }}^{R x}
\end{aligned}
$$

And lastly;

$$
P_{P D S C H}^{R x}=P_{P D S C H}^{T x}+G_{\text {ant }}^{T x}+G_{M I M O}^{T x}-P L-M-L_{\text {building }}-L_{\text {Body }}+G_{\text {ant }}^{R x}
$$

Where $P_{R S}^{R x}, P_{S S}^{R x}, P_{P B C H}^{R x}, P_{\text {control }}^{R x}$ and $P_{P D S C H}^{R x}$ are received power levels (in $\mathrm{dBm}$ ) of RS, SS, PBCH, control channels region and PDSCH, respectively, $G_{a n t}^{T x}$ is the antenna gain of transmitter (Tx), $G_{M I M O}^{T x}$ is MIMO gain of Tx and equals $10 \times \log \left(N_{\text {MIMO }}\right)$, where $N_{\text {MIMO }}$ is the number of MIMO antenna terminals, $P L$ is the pathloss between the transmitter and receiver, $M$ is the margin of interference plus shadowing, $L_{\text {building }}$ is the building penetration loss, $L_{B o d y}$ is the body loss and $G_{a n t}^{R x}$ is the antenna gain of receiver.

At this point, it is extremely important to understand the difference between the transmission antenna ports and the MIMO unit of an LTE eNodeB as they are not the same. Essentially, an antenna port is a new concept introduced 
by the $3 \mathrm{GPP}$ to act as a logical port that does not necessarily correspond to an antenna. The objective is to relieve the pressure from the user equipment to detect beamforming-centric transmission from an eNodeB by means of assisting signals (pilots). In other words, pilots (RS) are used when MIMO is used at the eNodeB to inform the user terminal on any beamforming operations being carried out at the transmitter (eNodeB) (3GPP TS 36.211, 2007; Sesia, Toufik, \& Baker, 2011). Furthermore, MIMO denotes multiple physical antennas usage at the eNodeB. However, for MIMO to work, logical antenna ports have to work as well to impart the number of physical MIMO antennas used by means of pilot signals, i.e. RS.

\section{PATHLOSS MODEL}

The propagation or path losses $P L$ used in Equations (12) through (16) are addressed by an enhanced Cost321-Hata model. The proposed model accounts for signal attenuation, clutter morphology, variable cell range and frequency ranges of (150-1500) MHz or (1500-2000) MHz as described in the following expression:

$$
\begin{aligned}
& P L=A+B \times \log (f)-13.82 \times \log \left(H_{T x}\right)-a\left(h_{R x}\right)+ \\
& \Psi \times \log (D)+L_{\text {clutter }}
\end{aligned}
$$

where $A$ and $B$ are both constants, and their values are shown in Table 2 (Medeisis \& Kajackas, 2000):

Table 2.

Values of Constants $A$ and $B$

\begin{tabular}{lcc}
\hline Frequency $(\mathrm{MHz})$ & A & B \\
\hline $150-1500$ & 69.55 & 26.16 \\
$1500-2000$ & 46.3 & 33.9 \\
\hline
\end{tabular}

$f$ is the transmission frequency of the transmitter (in $\mathrm{MHz}$ ), $H_{T X}$ is transmitter height (in meters), $D$ is the distance between the transmitter and receiver (in $\mathrm{Km})$, and $a\left(h_{R x}\right), S$ and $L_{\text {clutter }}$ are described as follows:

$a\left(h_{R x}\right)$ is the receiver height correction factor (in meters):

$$
a\left(h_{R x}\right)=\left\{\begin{array}{lc}
3.2 \times\left(\log \left(11.75 H_{R x}\right)\right)^{2}-4.97 & \text { For dense urban \& urban } \\
H_{R x}(1.1 \log (f)-0.7)-(1.56 \log (f)-0.8) & \text { For suburban \& rural }
\end{array}\right.
$$




$$
\Psi= \begin{cases}44.9-6.55 \log \left(H_{T x}\right) & \text { For } D \geq 1 \mathrm{Km} \\ \frac{47.88+13.9 \log (f)-13.82 \log \left(H_{T x}\right)}{1.699} & \text { For } D<1 \mathrm{Km}\end{cases}
$$

And

$$
L_{\text {clutter }}= \begin{cases}3 & \text { For dense urban } \\ 0 & \text { For urban } \\ -2(\log (f / 28))^{2}-5.4 & \text { For suburban } \\ -4.78(\log (f))^{2}+18.33 \log (f)-40.94 & \text { For open rural } \\ -4.78(\log (f))^{2}+18.33 \log (f)-35.94 & \text { For quasi rural }\end{cases}
$$

\section{RESULTS AND DISCUSSIONS}

Based on the proposed methodology, this section provides the research analytical results that show the impact of overheads as well as individual transmission and reception powers of each considered physical download signal and channels.

\section{Common channels and signals overhead}

Figure 3 depicts the amount of overhead imposed by common channels and signals on shared (traffic) channels when $1.4 \mathrm{MHz}$ bandwidth is employed by an LTE transmitter, i.e. eNodeB. Clearly, the physical resources (resource elements) taken up by shared channels (PDSCH) are the highest in population among all channels and signals. However, payload carriers are inevitably disrupted to a certain extent, by control channels resource elements that affect negatively the number of user data carriers. From the perspective of synchronization signals and broadcast channels, their corresponding overhead is highly comparable and appears to be the smallest among all other common channels and signals due to their limited physical resources consumption that is constant for SS over any bandwidth and slightly less for PBCH due to the overlap with reference signals resource elements.

For the same number of antenna ports and sym, the control region overhead is remarkably the same, thus being bounded by the amount of overlap with RS resources and control region width, i.e. sym in Eq.(8). 
Similarly, RS overhead remains unchanged for the same number of antenna ports. However, the peculiarity of RS's silent resource elements gives rise to extra overhead, as seen in Figure 2.

Lastly, the effect of the higher number of antenna ports at the transmitter on the amount of overhead imposed by common channels and signals on useful user data is illustrated in Figure 4 whereby the higher the number of antenna ports used, the greater the amount of overhead. Moreover, the smaller the bandwidth (RBs), the more serious impact the overhead imposes.

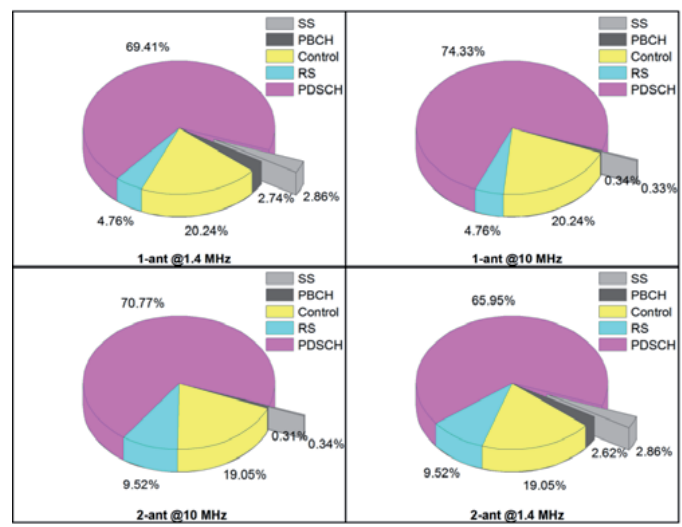

Figure 3. Physical downlink channels and signals overhead for the LTE eNodeB transmission.

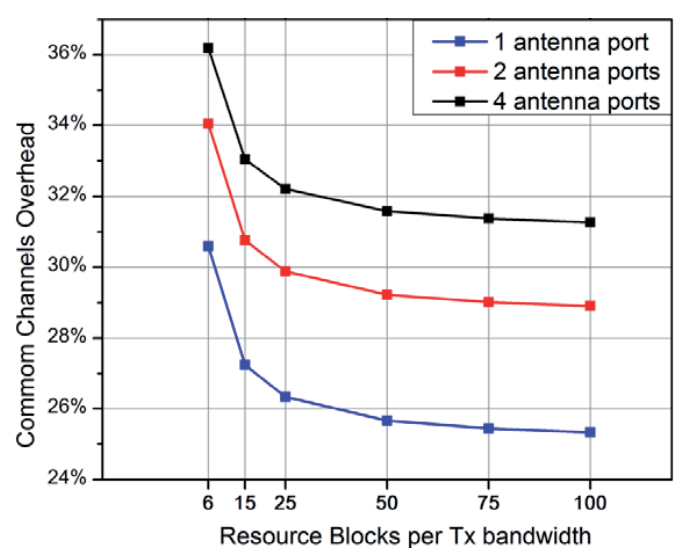

Figure 4. Total overhead associated with common channels and signals with respect to the number of transmitted resource blocks for 1, 2 And 4 transmit antenna ports. 


\section{Common Channels and Signal Powers}

The power transmitted by the eNodeB of inputs and propagation conditions shown in Table 2 is calculated with respect to various antenna ports numbers, as illustrated in Figure 5. Based on the above, the received power corresponding to each control channel and signal is determined as depicted in Figure 5. Under the same propagation circumstances and for $1.4 \mathrm{MHz}$ system bandwidth, channels and signals transmitted and received, powers tend to decrease linearly with the increased number of antenna ports. This is essential because the corresponding antenna port power significantly decreases in the order of 40,20 and $10 \mathrm{~W}$ for 1, 2 and 4 antenna ports respectively, due to the power sharing scheme. However, the RS signals shows an exceptional increase in their received powers as the antenna ports increase in number due to the added MIMO gain. Ultimately, the power allocation among subcarriers means that if the coverage is restricted by the transmitted power, the operator should increase the output power or decrease the channel bandwidth.

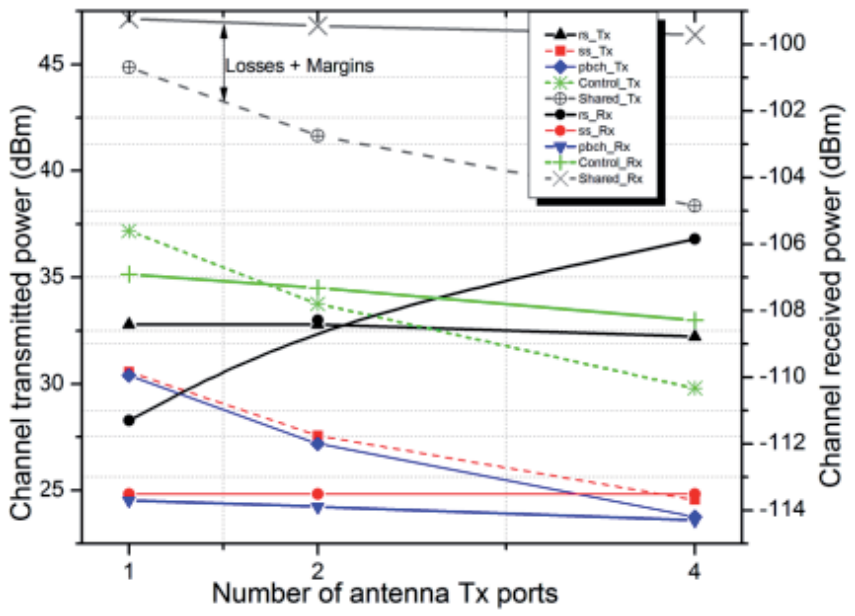

Figure 5. Common channels and signals transmitted and received power for different number of antenna transmission ports.

\section{CONCLUSION}

In view of the whole new bandwidth-agnostic $4 \mathrm{G}$ networks in general and LTE systems in particular, the link budget, capacity and coverage dimensioning calculations of such networks turn to be more intricate than that of legacy 
systems due to the peculiarities the LTE physical channels and signals possess during transmission and reception. In this paper, the resource element level computations of the LTE downlink physical layer channels and signals have been addressed in detail. The calculations proposed in this paper are quite beneficial for low and high level dimensioning procedures of LTE networks. it has been shown in this study that the cell-specific reference signal (pilot) overhead depends on the number of active antenna ports. Furthermore, from the overhead and power consumption standpoint, silent reference signals resource elements do impose extra overhead on the user payload and thus consume additional power. However, from the channel estimation and transmission point of view, they don't transmit any power. Instead they lend their powers to their corresponding active or used RS resources and thus act as power boosting entities. To sum up, downlink control channels and reference signals are the most important factors affecting dimensioning and capacity results. The rest of the downlink physical channels and signals can be ignored. notably, and it is recommended that for future work, this work can be expanded to include the dimensioning methodology of LTE uplink. This, however, is different from the LTE downlink formulas in that it is involves the transmission and reception of A slightly different multiplexing scheme, i.e. SC-FDMA (Single Frequency Division Multiple Access), which in turn comprises different physical resources allocations and structures.

\section{REFERENCES}

3GPP TS 25.211. (2002). Physical channels and mapping of transport channels onto physical channels (FDD) (Release 99).

3GPP TS 25.221. (2002). Physical channels and mapping of transport channels onto physical channels (TDD).

3GPP TS 25.942. (2008). Evolved Universal Terrestrial Radio Access Network (E-UTRAN); Radio Frequency (RF) system scenarios (Release 8).

3GPP TS 36.211. (2007). Evolved Universal Terrestrial Radio Access (E-UTRA); Physical channels and modulation.

3GPP TS 36.300. (2006). Evolved Universal Terrestrial Radio Access (E-UTRA) and Evolved Universal Terrestrial Radio Access Network (E-UTRAN); Overall description; Stage 2.

3GPP TS 36.410. (2010). Evolved Universal Terrestrial Radio Access Network (E-UTRAN); S1 general aspects and principles. 
Ball, C., Hindelang, T., \& Kambourov, I. (2008). Spectral efficiency assessment and radio performance comparison between LTE and WiMAX. Indoor and Mobile Radio, 0-5.

Basit, A. (2009). Dimensioning of LTE: Network description of models and tool, coverage and capacity estimation of 3 GPP Long Term Evolution radio interface. Evolution. Helsinki University of Technology.

Cui, D. (2009). LTE peak rates analysis. Paper presented at Conference. WOCC 2009 18th Annual, 3-5.

Kattan, A. (2009). Building distributed heterogeneous smart phone Java applications an evaluation from a development perspective. Journal of Information and Communication Technology, 8, 67-83.

Medeisis, a., \& Kajackas, A. (2000). On the use of the universal OkumuraHata propagation prediction model in rural areas. VTC2000-Spring. IEEE 51st Vehicular Technology Conference Proceedings (Cat. No.00CH37026), 3, 1815-1818. IEEE.

Ratasuk, R., Ghosh, A., Love, R., Nory, R., \& Classon, B. (2008). TDD design for UMTS Long-Term Evolution. IEEE 19th International Symposium on Personal, Indoor and Mobile Radio Communications, 1-5. doi:10.1109/PIMRC.2008.4699646

Rezaei, F. (2010). A comprehensive analysis of LTE physical layer. University of Nebraska - Lincoln.

Rezaei, F., Hempel, M., \& Sharif, H. (2011). LTE PHY performance analysis under 3GPP standards parameters. Computer Aided Modelling and Design of Communication Links and Networks (CAMAD). IEEE 16th International Workshop (pp. 102-106).

Sanchez, J. J., Morales-Jimenez, D., Gomez, G., \& Enbrambasaguas, J. T. (2007). Physical layer performance of long term evolution cellular technology. 16th IST Mobile and Wireless Communications Summit (pp. 1-5). doi:10.1109/ISTMWC.2007.4299090

Sesia, S., Toufik, I., \& Baker, M. (2011). LTE - The UMTS long term evolution: From theory to practice. John Wiley and Sons.

The Global mobile Suppliers Association (GSA). (2011a). Evolution to LTE Report. http://www.gsacom.com/gsm_3g/info_papers.php4 
The Global Mobile Suppliers Association (GSA). (2011b). Status of the LTE ecosystem. http://www.gsacom.com/gsm_3g/info_papers.php4. Group

The Global Mobile Suppliers Association (GSA). (2011c). LTE operator commitments. http://www.gsacom.com/gsm_3g/info_papers.php4

The Global Mobile Suppliers Association (GSA). (2011d). Mobile broadband fact sheet. http://www.gsacom.com/gsm_3g/info_papers.php4 
